\title{
Are malnourished patients getting the attention they require? Results of a cross-sectional survey from a teaching hospital in London
}

\author{
A. Fikree, A. Kramer, A. Prabhakar, C. Soulsby, J. Powell-Tuck and M. Glynn \\ Department of Gastroenterology, Royal London Hospital, London E1 1BB, UK
}

Twenty-eight percent of patients who are admitted to hospital are malnourished ${ }^{(1)}$. As a response to this several nutritional screening guidelines have been created to identify malnutrition and prevent $\mathrm{it}^{(2)}$. Despite this, studies have shown that the rates of screening remain far from perfect ${ }^{(3)}$. Our study aimed to assess whether the rates of screening and monitoring for the subgroup of malnourished patients, which is the group the guidelines are trying to target, is better than the rate of screening for all patients.

A cross-sectional audit was carried out at the Royal London Hospital on 28 March 2009. Out of 469 adult patients in the hospital that day, 279 patients $(60 \%)$ were on the acute and general medical and surgical wards. All 279 had their notes, drug charts and observation charts assessed for the presence of nutritional screening, monitoring and the provision of nutritional supplementation. Patients were then asked whether they had any unintentional weight loss (UWL) prior to admission and whether they were hungry. Thirty-six patients could not report due to not being by bed, language barriers or being medically unwell, leaving 243 patients (87\%) who self-reported.

Ninety-nine $(41 \%)$ of the 243 patients reported UWL, 2 of these had a BMI $<18.5$. One other patient who could not self-report had a BMI of 18 . Hence 100 patients were considered malnourished.

Table 1 compares the rates of nutritional screening, monitoring and nutritional supplementation in malnourished patients with all patients.

\begin{tabular}{lcc}
\hline & Malnourished patients - 100 (number, \%) & All patients - 279 (number, \%) \\
\hline Weight present & 39,39 & 109,39 \\
Height and weight present & 8,8 & 30,11 \\
BMI recorded & 4,4 & 13,5 \\
Food chart present & 19,19 & 59,21 \\
Food chart up to date & 14,14 & 49,18 \\
Nutrition screening chart present & 27,27 & 95,34 \\
Nutrition screening chart up to date & 15,15 & 56,20 \\
Oral supplementation (OS) given & 18,18 & 43,15 \\
Enteral nutrition given & 2,2 & 15,5 \\
Parenteral nutrition given & 1,1 & 4,1 \\
OS and up to date food chart & 6,33 & 16,37 \\
OS and up to date nutritional screening chart & 4,22 & 14,32 \\
Reported hunger & 36,36 & 60,25 \\
\hline
\end{tabular}

Compared to all patients, the malnutrition group was less likely to have screening and monitoring; they were more likely to have oral nutritional supplementation (ONS) and feel hungry. Even the patients with malnutrition who were on ONS were less likely to be monitored by way of an up to date food chart and nutritional screening chart.

In a busy clinical environment, it is sometimes said, against hospital and national guidelines, that nutritional screening needs to be reserved on a common sense basis, for those who appear to have a nutritional problem. This in effect inserts an "eye-balling" pre-screen process which we do not agree with. However, these data show that in fact screening is often not conducted even in patients clearly at high risk. If we remain bad at identifying and managing even high risk groups then we have no hope of preventing problems of malnutrition. A culture change is imperative before this can be achieved.

1. BAPEN Nutrition Screening Survey in the UK in 2008 (ISBN 9781899467419 ).

2. Nutritional support in adults: oral nutrition support, enteral tube feeding and parenteral nutrition NICE 2006. http://guidance.nice.org.uk/CG32/ Guidance/pdf/English

3. Campbell SE, Avenall A \& Walker AE (2002). Q J Med 55, 83-87. 